\title{
Instability of coupled gravity-inertial-Rossby waves on a $\beta$-plane in solar system atmospheres
}

\author{
J. F. McKenzie \\ Astrophysics and Cosmology Research Unit, School of Mathematical Sciences, University of KwaZulu-Natal, Durban, 4041, \\ South Africa \\ Department of Physics, CSPAR, University of Alabama, AL, USA \\ Senior Member, King's College, Cambridge, UK \\ Received: 4 August 2009 - Revised: 28 September 2009 - Accepted: 28 October 2009 - Published: 9 November 2009
}

\begin{abstract}
This paper provides an analysis of the combined theory of gravity-inertial-Rossby waves on a $\beta$-plane in the Boussinesq approximation. The wave equation for the system is fifth order in space and time and demonstrates how gravity-inertial waves on the one hand are coupled to Rossby waves on the other through the combined effects of $\beta$, the stratification characterized by the Väisälä-Brunt frequency $N$, the Coriolis frequency $f$ at a given latitude, and vertical propagation which permits buoyancy modes to interact with westward propagating Rossby waves. The corresponding dispersion equation shows that the frequency of a westward propagating gravity-inertial wave is reduced by the coupling, whereas the frequency of a Rossby wave is increased. If the coupling is sufficiently strong these two modes coalesce giving rise to an instability. The instability condition translates into a curve of critical latitude $\Theta_{c}$ versus effective equatorial rotational Mach number $M$, with the region below this curve exhibiting instability. "Supersonic" fast rotators are unstable in a narrow band of latitudes around the equator. For example $\Theta_{c} \sim 12^{\circ}$ for Jupiter. On the other hand slow "subsonic" rotators (e.g. Mercury, Venus and the Sun's Corona) are unstable at all latitudes except very close to the poles where the $\beta$ effect vanishes. "Transonic" rotators, such as the Earth and Mars, exhibit instability within latitudes of $34^{\circ}$ and $39^{\circ}$, respectively, around the Equator. Similar results pertain to Oceans. In the case of an Earth's Ocean of depth $4 \mathrm{~km}$ say, purely westward propagating waves are unstable up to $26^{\circ}$ about the Equator. The nonlinear evolution of this instability which feeds off rotational energy and gravitational buoyancy may play an important role in atmospheric dynamics.
\end{abstract}

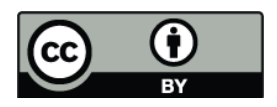

Correspondence to: J. F. McKenzie (mckenziej@ukzn.ac.za)
Keywords. Meteorology and atmospheric dynamics (Middle atmosphere dynamics)

\section{Introduction}

It is well known that rotating planetary atmospheres permit the propagation of different types of wave modes. For example gravity-inertial waves propagate anisotropically with dispersion for frequencies above the Coriolis frequency, $f$, and below the Väisälä-Brunt frequency $N$ (see for example Eckart, 1960; Lighthill, 1980). Rossby waves, which propagate at lower frequencies $(\omega<f)$, are characterized by mainly horizontal fluid motions in which the total vertical vorticity (fluid plus planet) is conserved along a northward moving fluid element and arise because of the $\beta$-effect describing the latitudinal variation of the vertical Coriolis frequency (see for example Pedlosky, 1987; Gill, 1982). Here we present a combined theory of gravity-inertial and Rossby waves and show that if the coupling constant is sufficiently strong the wave system can be driven unstable. The instability arises from the interaction between westward propagating gravity-inertial waves and Rossby waves which can release the energy stored in gravitational buoyancy and rotational energy. As we shall see below the instability condition predicts that for a given effective equatorial rotational Mach number, waves at latitudes less than critical are unstable. For fast rotators the instability is confined to a narrow belt of latitudes around the equator whereas slow rotators exhibit instability at all latitudes except near the poles.

This study does not, of course, claim to be the first to investigate the properties of linear coupled gravity-inertialRossby waves. The crucial wave equation for the system (see Eqs. 11 and 18 below) appears in, for example, the classical

Published by Copernicus Publications on behalf of the European Geosciences Union. 
text by Pedlosky (1987), where his Eq. (3.6.9) is identical except that topography assumes a role similar to the $\beta$-effect. However, the novelty of the present work is the examination of the nature of the roots of the corresponding dispersion equation for values of the coupling parameter where the dynamics of the two modes overlap in both frequency and wavenumber. This reveals a convective instability of the coupled system. Equatorial regions, where the Coriolis frequency $f$ goes to zero, require special attention on account of the development of a waveguide system (Moore and Philander, 1977; Cane and Sarachik, 1976).

The instability described here appears to be a new result which may be of some importance in atmosphere-ocean dynamics. The instability arises from the coupling between two different modes whose frequencies overlap in certain wave number regimes if the coupling is sufficiently strong. We emphasize however that the present instability is not the same as that studied by Sakai (Sakai, 1989) where a Rossby-Kelvin instability arises by virtue of the resonant interaction between a Rossby and a Kelvin wave in a two layered latitudinally sheared zonal flow. In this latter case instability is due to the interaction between positive and negative energy waves on either side of a vortex sheet brought about by Doppler shifting of the wave frequencies on either side.

In Sect. 2 we derive the wave equation for coupled gravityinertial-Rossby waves in a rotating, stratified atmosphere using the $\beta$-plane approximation (which simplifies the spherical geometry whilst retaining the essential dynamics) and the Boussinesq approximation which filters out higher frequency acoustic waves. This coupled wave equation is fifth order in space and time (second in space and third in time). The corresponding "local" dispersion relation for plane waves is analyzed in Sect. 3. It is a cubic equation for the frequency $\omega$ for given wave number vector $\boldsymbol{k}$ in which the three roots represent an eastward propagating gravity-inertial mode (modified somewhat by the $\beta$ effect) and two westward propagating modes. In these latter coupled modes the frequency of the Rossby wave is increased whereas the frequency of the gravity-inertial mode is decreased. If the coupling is sufficiently strong these two modes coalesce and the system is driven unstable. In Sect. 4 we apply this stability condition to Solar System Atmospheres (the Planets and the Sun). In terms of the stability condition, in the parameter space of a critical latitude versus an effective equatorial rotational Mach number, we find that the outer gaseous giants are "supersonic" rotators with instability confined to a fairly narrow belt of latitudes around the equator. For example in the case of Jupiter the instability is confined to within about $12^{\circ}$ of the equator. It must of course be borne in mind that the equatorial region requires special treatment as already noted. On the other hand slow "subsonic" rotators (Mercury, Venus and the Sun) exhibit instability at all latitudes except very close to the poles. The Earth is slightly supersonic with a band of latitudes up to $34^{\circ}$ of the equator subject to instability, whilst Mars appears to be transonic with its unstable belt stretching to $39^{\circ}$ around its equator. We note that Oceans also exhibit this type of instability. As an example of an Earth's Ocean, of depth $4 \mathrm{~km}$ yielding a shallow water speed of $200 \mathrm{~m} / \mathrm{s}$, the equatorial rotational Froude number is 2.3 which yields an unstable band of latitudes around the equator $26^{\circ}$ in width.

\section{Combined theory of gravity-inertial-Rossby waves on a $\beta$ plane}

We consider a background atmosphere stratified hydrostatically according to

$\frac{d p_{o}}{d z}=-\rho_{o} g$.

At a given latitude $\theta$ the vertical component of the planet's angular frequency $\Omega_{z}$ is given by

$\Omega_{z}=\Omega \sin \theta$

We make the $\beta$-plane approximation in which the latitudinal variation of the Coriolis frequency $2 \Omega_{z}$ about a given latitude $\theta_{o}$ is described (Pedlosky, 1987; Gill, 1982) by

$f=2 \Omega \sin \theta \approx f_{o}+\beta y$,

where

$f_{o}=2 \Omega \sin \theta_{o}, \quad \beta=\frac{2 \Omega \cos \theta_{o}}{R}$.

Here $R$ is the radius of the planet, $y=R \delta \theta$ is a local Cartesian northward coordinate, and $x$ is the eastward coordinate on the $\beta$-plane drawn tangent to the surface at $\theta_{o}$. The linearized equations of motion, with the Coriolis acceleration, are

$$
\begin{aligned}
\rho_{o}\left(\frac{\partial u_{x}}{\partial t}-f u_{y}\right) & =-\frac{\partial p_{e}}{\partial x}, \\
\rho_{o}\left(\frac{\partial u_{y}}{\partial t}+f u_{x}\right) & =-\frac{\partial p_{e}}{\partial y}, \\
\rho_{o} \frac{\partial u_{z}}{\partial t} & =-\frac{\partial p_{e}}{\partial z}-\rho_{e} g,
\end{aligned}
$$

for the perturbed velocity $\underline{u}$, pressure $p_{e}$ and density $\rho_{e}$. We make the Boussinesq approximation in which the variations in perturbation $\rho_{e}$ are quasi-incompressible in the sense that continuity approximates to

$$
\frac{\partial}{\partial z}\left(\rho_{o} u_{z}\right)=-\rho_{o}\left(\frac{\partial u_{x}}{\partial x}+\frac{\partial u_{y}}{\partial y}\right),
$$

whilst retaining the buoyancy force $\rho_{e} g$ in the vertical equation of motion. In addition vertical motions are buoyantadiabatic with (see Eckart, 1960; Lighthill, 1980),

$$
g \frac{\partial \rho_{e}}{\partial t}=N^{2}\left(\rho_{o} u_{z}\right)
$$


in which $N^{2}$, the square of the Väisälä-Brunt frequency, is given by

$N^{2}=-g\left(\frac{\rho_{o}^{\prime}(z)}{\rho_{o}(z)}-\frac{g}{c_{o}^{2}}\right), \quad c_{o}^{2}=\frac{\gamma p_{o}}{\rho_{o}}$.

We shall assume the atmosphere is stably stratified with $N^{2}>0$. In effect the Boussinesq approximation ignores variations in perturbation $\rho_{e}$, unless it is multiplied by $g$, and therefore filters out the higher frequency acoustic waves. Equations (5), (6) and (7) combine to yield

$$
\frac{\partial^{3} p_{e}}{\partial z^{2} \partial t}=\left(\frac{\partial^{2}}{\partial t^{2}}+N^{2}\right)\left(\frac{\partial q_{x}}{\partial x}+\frac{\partial q_{y}}{\partial y}\right)
$$

in which $q=\rho_{o}\left(u_{x}, u_{y}, u_{z}\right)$ is the perturbed mass flux vector. Hence Eq. (9) can be used in Eqs. (3) and (4), operated upon by $\partial^{3} / \partial z^{2} \partial t$, to eliminate $p_{e}$ and yield the following coupled equations for $q_{x}$ and $q_{y}$, namely,

$$
\begin{array}{r}
{\left[\frac{\partial^{4}}{\partial z^{2} \partial t^{2}}+\left(\frac{\partial^{2}}{\partial t^{2}}+N^{2}\right) \frac{\partial^{2}}{\partial x^{2}}\right] q_{x}=} \\
f \frac{\partial^{3} q_{y}}{\partial z^{2} \partial t}-\left(\frac{\partial^{2}}{\partial t^{2}}+N^{2}\right) \frac{\partial^{2} q_{y}}{\partial x \partial y}, \\
{\left[\frac{\partial^{4}}{\partial z^{2} \partial t^{2}}+\left(\frac{\partial^{2}}{\partial t^{2}}+N^{2}\right) \frac{\partial^{2}}{\partial y^{2}}\right] q_{y}=} \\
-f \frac{\partial^{3} q_{x}}{\partial z^{2} \partial t}-\left(\frac{\partial^{2}}{\partial t^{2}}+N^{2}\right) \frac{\partial^{2} q_{x}}{\partial x \partial y} .
\end{array}
$$

Obvious operations on, for example, Eq. (10b) to eliminate $q_{x}$, using Eq. (10a), and noting that $d f / d y=\beta$ from Eq. (2b), and factoring out $\partial^{3} / \partial z^{2} \partial t$, yields the wave equation for $q_{y}$, namely

$$
\begin{aligned}
& \frac{\partial}{\partial t}\left\{\frac{\partial^{2}}{\partial z^{2}}\left(\frac{\partial^{2}}{\partial t^{2}}+f^{2}\right)+\left(\frac{\partial^{2}}{\partial t^{2}}+N^{2}\right) \times\right. \\
& \left.\left(\frac{\partial^{2}}{\partial x^{2}}+\frac{\partial^{2}}{\partial y^{2}}\right)\right\} q_{y}=-\beta\left(\frac{\partial^{2}}{\partial t^{2}}+N^{2}\right) \frac{\partial q_{y}}{\partial x} .
\end{aligned}
$$

This equation describes the coupling between the inertial gravity waves on the one hand and Rossby waves on the other. Note that in the limit $\beta=0$ the zero of the curly bracket on the left hand side of Eq. (11), namely,

$$
\begin{array}{r}
\left\{\frac{\partial^{2}}{\partial z^{2}}\left(\frac{\partial^{2}}{\partial t^{2}}+f_{o}^{2}\right)+\left(\frac{\partial^{2}}{\partial t^{2}}+N^{2}\right) \times\right. \\
\left.\left(\frac{\partial^{2}}{\partial x^{2}}+\frac{\partial^{2}}{\partial y^{2}}\right)\right\} q_{y}=0
\end{array}
$$

is the classic gravity-inertial wave equation (see Eckart, 1960; Lighthill, 1980). Similarly in the limit $N^{2} \rightarrow \infty$ we have the two-dimensional Rossby wave equation (see Pedlosky, 1987; Gill, 1982),

$\frac{\partial}{\partial t}\left(\frac{\partial^{2}}{\partial x^{2}}+\frac{\partial^{2}}{\partial y^{2}}\right) q_{y}=-\beta \frac{\partial q_{y}}{\partial x}$.

Assuming $N^{2}$ is constant and north-south length scales $y$ very much less than $R \tan \theta_{0}$, the wave Eq. (11) admits JWKB plane wave solutions

$\frac{1}{k_{y}^{1 / 2}}\left[\exp \left( \pm i \int k_{y} d y\right)\right] \exp \left[i\left(\omega t-k_{x} x-k_{z} z\right)\right]$,

in which the north-south wave number $k_{y}$ satisfies the "local" dispersion equation, written in the form of a wave normal surface (Eckart, 1960), namely,

$-\frac{\left(\omega^{2}-f^{2}\right)}{\left(N^{2}-\omega^{2}\right)} k_{z}^{2}+k_{y}^{2}+\left(k_{x}^{2}+\frac{\beta}{2 \omega}\right)^{2}=\left(\frac{\beta}{2 \omega}\right)^{2}$.

In fact for $y \ll R \tan \theta_{0}, f$ may be replaced by $f_{0}$, the central value of $f$ at the latitude $\theta_{0}$, in Eq. (11). If $\omega<f$, this represents an ellipsoid in ( $\boldsymbol{k}$ space) of revolution about an axis parallel to the $k_{y}$ axis, but displaced by $-\beta / 2 \omega$ units along the $k_{x}$ axis. It may be viewed as providing the two-dimensional Rossby wave normal circle (Charney and Drazin, 1961), namely,

$k_{y}^{2}+\left(k_{x}+\frac{\beta}{2 \omega}\right)^{2}=\left(\frac{\beta}{2 \omega}\right)^{2}$

with a vertical propagation structure. Similarly, if $N>\omega>$ $f$, Eq. (15) represents a hyperboloid which describes the Rossby $\beta$ effect on inertial-gravity waves which, in the absence of $\beta$, describes propagation on a cone. These surfaces are particularly useful in ray theory within the JWKB approximation appropriate to weakly inhomogeneous media.

It is of interest to note that a similar analysis can be carried out for an ocean of depth $h$ using shallow water theory (see for example Stoker, 1965). In this case the perturbed elevation $\eta$ of the surface wave enters the linearized continuity equation and horizontal momentum equations in the form

$$
\begin{aligned}
& \frac{\partial \eta}{\partial t}+h\left(\frac{\partial u_{x}}{\partial x}+\frac{\partial u_{y}}{\partial y}\right)=0, \\
& \frac{\partial u_{x}}{\partial t}-f u_{y}=-g \frac{\partial \eta}{\partial x}, \quad \frac{\partial u_{y}}{\partial t}+f u_{x}=-g \frac{\partial \eta}{\partial y},
\end{aligned}
$$

in which the perturbation pressure $p_{e}$ is in hydrostatic pressure balance at the surface, i.e. $p_{e}=-\rho_{o} g \eta$. Equations (17) and (18) yield the following wave equation for the surface elevation $\eta$ or $\left(u_{y}\right)$, namely,

$\frac{\partial}{\partial t}\left[\frac{\partial^{2}}{\partial t^{2}}+f^{2}-g h\left(\frac{\partial^{2}}{\partial x^{2}}+\frac{\partial^{2}}{\partial y^{2}}\right)\right] u_{y}=\beta g h \frac{\partial u_{y}}{\partial x}$. 


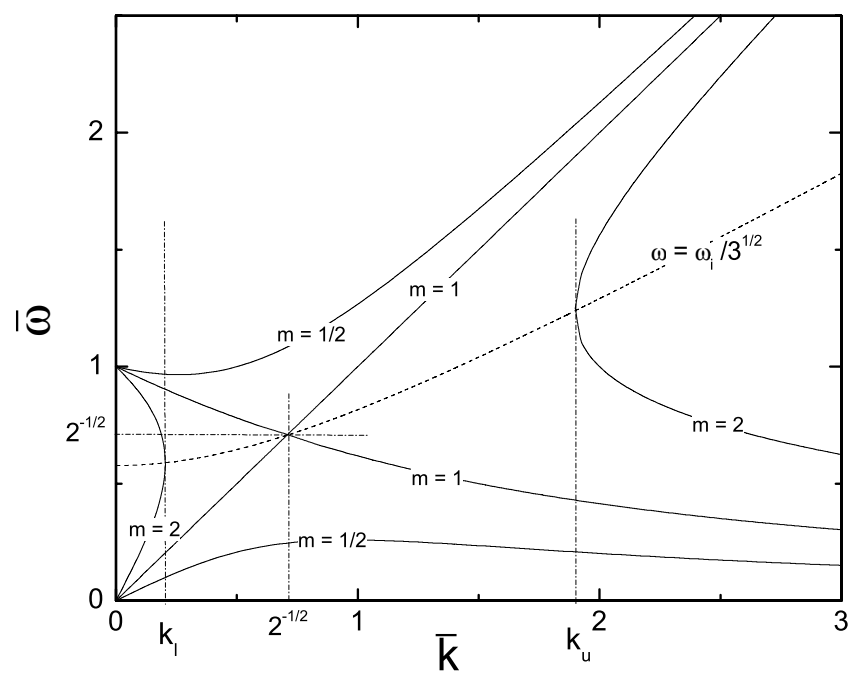

Fig. 1. The diagnostic diagram, $(\bar{\omega}, \bar{k})$ plot, for the coupled modes, including a stable case $(m=1 / 2)$, an unstable case $(m=2)$, and the critical case $(m=1)$. The higher frequency curve segments (above the locus $\omega=\omega_{i} / \sqrt{3}$ ) describe the gravity-inertial mode, and the lower frequency segments the Rossby mode. The coupling of these modes leads to a convective instability for $m>1$.

The corresponding dispersion equation, which combines two-dimensional inertial waves with Rossby waves in shallow water may be written

$\omega\left(\omega^{2}-\omega_{i}^{2}\right)=\beta V^{2} k \cos \phi$,

in which

$\omega_{i}^{2}=f^{2}+V^{2} k^{2}, \quad V^{2}=g h$,

and $k_{x}=k \cos \phi$, so that westward propagation implies $\cos \phi<0$.

\section{The general dispersion equation: stability analysis}

Here our main aim is to examine the stability of the system, for which purpose we re-write Eq. (15) as one determining the frequency, $\omega$, as a function of $\boldsymbol{k}$. For frequencies $\omega \ll$ $N$ Eq. (15) may be cast in the form of the shallow water dispersion Eq. (20) in which the wave speed $\sqrt{g h}$ is replaced by its atmospheric counterpart (Mekki and McKenzie, 1977), i.e.,

$V=\frac{N}{k_{z}}$.

Normalizing $\omega$ with respect to $f$, and $k=\sqrt{k_{x}^{2}+k_{y}^{2}}$ with respect to $f / V$, the dimensionless form of the dispersion equation is $\bar{\omega}\left(\bar{\omega}^{2}-\bar{\omega}_{i}^{2}\right)=-m \bar{k}$,

$\bar{\omega}_{i}^{2}=1+\bar{k}^{2}$,

$m=\left(\frac{\beta V}{f^{2}}\right)|\cos \phi|, \quad \overline{\boldsymbol{k}}=\bar{k}(\cos \phi, \sin \phi)$.

In the definition of the coupling parameter, $m$, we have taken the modulus of $\cos \phi$ and reversed the sign in Eq. (23) to indicate that we consider waves propagating in the second or third quadrant corresponding to westward propagation for $\omega>0$. Equation (23) readily lends itself to the graphical solution, as shown in Fig. 1, in which the roots are given as intersections between the left hand side plotted as a function of $\bar{\omega}$ and the constant, $-m \bar{k}$, represented by a horizontal line. It is clear that if $|m| k<2 \omega_{i}^{3} /(3 \sqrt{3})$, the latter being the modulus of the minimum of the left hand side occurring at $\omega_{m}=\omega_{i} / \sqrt{3}$, there are two positive real roots for $\omega$, corresponding to westward propagation $\cos \phi<0$, and a third negative real root corresponding to eastward propagation of an inertial-gravity wave at a frequency greater than its "normal" value $\left|-\omega_{i}\right|$.

The two positive roots for $\omega$, describing the combined effects of $\beta$ and $N$, represent a Rossby wave with its frequency increased and an inertial-gravity wave with its frequency reduced. As the coupling $|m| k$ increases the roots move toward each other and coalesce when the line touches the minimum of the left hand side. A further increase in $|m| \bar{k}$ leads to complex conjugate roots

$\bar{\omega}-\bar{\omega}_{m}=\frac{ \pm i}{\sqrt{3 \omega_{m}}}\left[|m| k-\frac{2}{3} \frac{\omega_{i}^{3}}{\sqrt{3}}\right]^{\frac{1}{2}} \equiv \pm i \bar{\gamma}$.

Hence the system is unstable if

$|m|>\frac{2}{3 \bar{k} \sqrt{3}}\left(1+\bar{k}^{2}\right)^{\frac{3}{2}} \equiv F(\bar{k})$.

Since $F(\bar{k})$ has a minimum value of 1 at $\bar{k}^{2}=1 / 2$ the instability condition is simply

$m>1$.

When condition (27a) is satisfied there is a band of unstable wave numbers lying between $\boldsymbol{k}_{l}$ and $\boldsymbol{k}_{u}$ where these are the roots of

$|m|=F(\bar{k})$.

The central frequency of the instability (occurring at $\bar{k}^{2}=$ $1 / 2$ ) is the local Coriolis frequency divided by $\sqrt{2}$, i.e. $f / \sqrt{2}$. The corresponding instability growth rate is given by $\bar{\gamma}=F(m-1)^{1 / 2} / \sqrt{3}$ and is shown as a function of $\bar{k}$, for different values of the coupling parameter $m>1$ in Fig. 2 . 


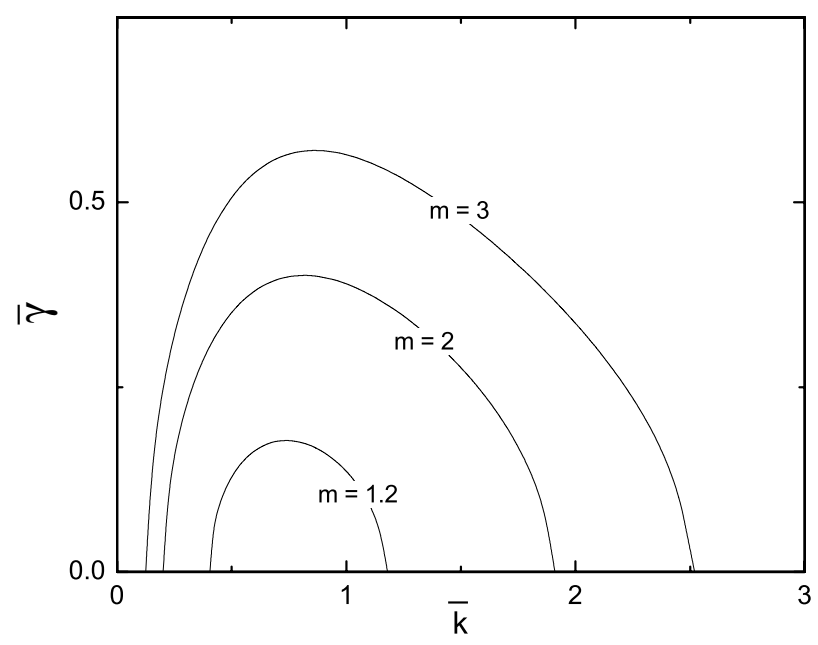

Fig. 2. The normalized growth rate $\bar{\gamma}$ as a function of $\bar{k}$ for $m=1.2$, 2 and 3 .

The instability condition $|m|>1$ can be interpreted in various ways, for example it can take the form

$\frac{\sin ^{2} \theta_{o}}{\cos \theta_{o}}<\frac{|\cos \phi|}{\left(k_{z} H\right)} \frac{\sqrt{g H}}{2(\Omega R)}$.

The angle $\phi$ is defined by Eq. (24), $H$ is the density scale height and $R$ is the radius of the body under study. Given the vertical wavelength, $k_{z} H$, and the planetary characteristics, ( $\sqrt{g H}$ and $\Omega R$ ), Eq. (28) predicts the angular width of the latitudinal band around the equator giving rise to unstable waves. We examine this interpretation in the next section with application to the Solar System.

\section{Application to solar system planets (and the Sun)}

The equivalent atmospheric shallow water speed $\sqrt{g H}$ is in fact very nearly the sound speed, $c$, in the atmosphere since scale height $H \simeq c^{2} / g$ (and $N \sim g / c$ ). Therefore the instability condition Eq. (28) may be written as

$\Theta_{o} \leq \sin ^{-1} \Theta_{c}$,

in which

$\sin ^{2} \Theta_{c}=\frac{\left(\sqrt{1+16 M^{2}}-1\right)}{8 M^{2}}$,

$M_{e q}=\frac{\Omega R}{c}, \quad M \equiv M_{e q}\left(\frac{k_{z} H}{\cos \phi}\right)$.

The Mach number $M_{e q}$ (ratio of the equatorial rotation speed to the sound speed) plays a crucial role in the instability condition, which is shown in Fig. 3 as the region below the curve of the critical latitude $\Theta_{c}$. The latter is plotted in Fig. 3 as a function of an effective Mach number, $M$, defined by

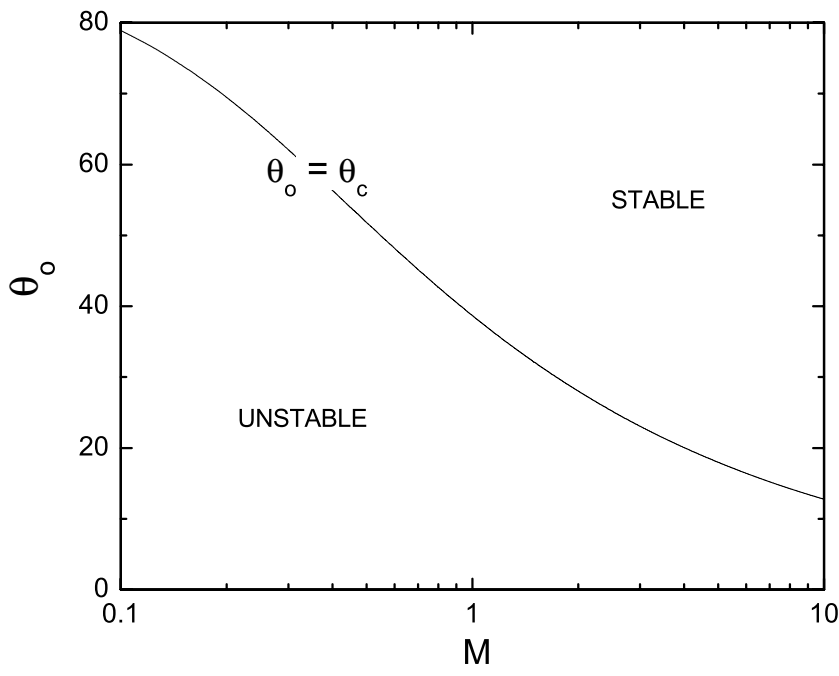

Fig. 3. The instability condition, relations (29), with the critical latitude $\theta_{c}$ as a function of the effective rotational Mach number, $M$, separating the parameter space into stable and unstable regions.

Eqs. (29b) and (29c), which combines the rotational Mach $M_{e q}$ with the configuration of the wave through the parameter $k_{z} H /|\cos \phi|$. In supersonically rotating planets $\left(M_{e q}>1\right)$ such as in the outer giants, the instability is confined to a fairly narrow band of latitudes around the equator, whereas subsonically rotating bodies (Sun, Mercury and Venus - the last with an astonishingly slow rotation in which a day is longer than a year) for which $M_{e q} \ll 1$, instability occurs at almost all latitudes, with the fastest growing mode near the equator. Table 1 presents a summary of planetary characteristics for the solar system, especially the equatorial Mach number $M_{e q}$, which yields the corresponding critical latitude $\Theta_{c}$ for instability. Note that Earth and Mars are "transonic" planets in which $\Theta_{c}$ is around $34^{\circ}$ and $39^{\circ}$, respectively. (The numbers given should be taken as "ball park" since they require detailed knowledge of the composition through the mean molecular weight $\mu$ and the temperature $T$.) In the case of an Ocean the instability condition takes the same form as Eq. (29) except that the effective Mach number $M$ is now given by

$M=\frac{M_{e q}}{|\cos \phi|}, \quad M_{e q}=\frac{\Omega R}{\sqrt{g h}}$,

in which $\sqrt{g h}$ is the shallow water wave speed. In an Earth's ocean of depth $4 \mathrm{~km}$ say, this speed is $200 \mathrm{~m} / \mathrm{s}$ so that $M_{e q}=2.3$. Therefore purely westward propagating waves $(|\cos \phi|=1)$ are unstable in a band of latitudes around the equator $26^{\circ}$ in width. 
Table 1. Properties of the planets and the Sun required in the calculation of the stability condition and the critical latitude $\Theta_{c}$.

\begin{tabular}{cccccc}
\hline Planet & $\Omega R$ & $\mathrm{~g}$ & $\mu$ & $\mathrm{T}$ & $c \sim \sqrt{g H}$ \\
\hline Mercury & $3.03 \mathrm{~m} / \mathrm{s}$ & $3.7 \mathrm{~m} / \mathrm{s}^{2}$ & 16 & $90-700^{\circ} \mathrm{K}$ & $220-570 \mathrm{~m} / \mathrm{s}$ \\
Venus & $2.00 \mathrm{~m} / \mathrm{s}$ & $8.9 \mathrm{~m} / \mathrm{s}^{2}$ & 43 & $400-735^{\circ} \mathrm{K}$ & $280-380 \mathrm{~m} / \mathrm{s}$ \\
Earth & $465 \mathrm{~m} / \mathrm{s}$ & $10 \mathrm{~m} / \mathrm{s}^{2}$ & 29 & $300^{\circ} \mathrm{K}$ & $350 \mathrm{~m} / \mathrm{s}$ \\
Mars & $242 \mathrm{~m} / \mathrm{s}$ & $3.7 \mathrm{~m} / \mathrm{s}^{2}$ & 32 & $230^{\circ} \mathrm{K}$ & $246 \mathrm{~m} / \mathrm{s}$ \\
Jupiter & $12.6 \mathrm{~km} / \mathrm{s}$ & $25 \mathrm{~m} / \mathrm{s}^{2}$ & 1.3 & $165^{\circ} \mathrm{K}$ & $1.13 \mathrm{~km} / \mathrm{s}$ \\
Saturn & $9.9 \mathrm{~km} / \mathrm{s}$ & $8.96 \mathrm{~m} / \mathrm{s}^{2}$ & 1.0 & $135^{\circ} \mathrm{K}$ & $1.33 \mathrm{~km} / \mathrm{s}$ \\
Uranus & $2.60 \mathrm{~km} / \mathrm{s}$ & $8.7 \mathrm{~m} / \mathrm{s}^{2}$ & 1.44 & $76^{\circ} \mathrm{K}$ & $0.8 \mathrm{~km} / \mathrm{s}$ \\
Neptune & $2.67 \mathrm{~km} / \mathrm{s}$ & $11.2 \mathrm{~m} / \mathrm{s}^{2}$ & 1.56 & $77^{\circ} \mathrm{K}$ & $0.77 \mathrm{~km} / \mathrm{s}$ \\
Sun & $2.00 \mathrm{~km} / \mathrm{s}$ & $274 \mathrm{~m} / \mathrm{s}^{2}$ & 1.0 & $6 \times 10^{3}-10^{6} \circ \mathrm{K}$ & $120-9.3 \mathrm{~km} / \mathrm{s}$ \\
\hline
\end{tabular}

\begin{tabular}{ccccc}
\hline Planet & $N$ & $H$ & $M_{e q}=\frac{\Omega R}{c}$ & $\Theta_{c}{ }^{\circ}$ \\
\hline Mercury & $0.017-0.006$ & $13-91 \mathrm{~km}$ & $0.014-0.005$ & $\sim 90^{\circ}$ \\
Venus & $0.031-0.023$ & $9 \mathrm{~km}$ & $0.007-0.005$ & $\sim 90^{\circ}$ \\
Earth & 0.03 & $12 \mathrm{~km}$ & 1.33 & $\sim 34^{\circ}$ \\
Mars & 0.015 & $16 \mathrm{~km}$ & 0.98 & $\sim 39^{\circ}$ \\
Jupiter & 0.022 & $51 \mathrm{~km}$ & 11.2 & $\sim 12^{\circ}$ \\
Saturn & 0.067 & $195 \mathrm{~km}$ & 7.4 & $\sim 14.8^{\circ}$ \\
Uranus & 0.011 & $74 \mathrm{~km}$ & 3.3 & $\sim 22^{\circ}$ \\
Neptune & 0.0145 & $53 \mathrm{~km}$ & 3.8 & $\sim 20^{\circ}$ \\
Sun & $0.023-0.03$ & $3 \times 10^{4}-180 \mathrm{~km}$ & $0.017-0.22$ & $\sim 87^{\circ}-68^{\circ}$ \\
\hline
\end{tabular}

\section{Summary}

We have shown that if the coupling parameter $m>1$ the combined system of linear gravity-inertial-gravity-Rossby waves is driven unstable by the interaction between the westward propagating gravity-inertial mode (whose frequency is lowered by the coupling) and a Rossby wave (whose frequency is raised to match that of the gravity-inertial mode). The instability condition Eq. (27a) or relations (29) is shown in Fig. 3 which depicts the unstable/stable regions, in the latitude $\Theta_{o}$, Mach number $M$ parameter space. It shows that fast rotators $(M>1)$ are only unstable in a fairly narrow band around the equator whereas slow rotators $(M<1)$ are unstable at all latitudes except near the poles. It may be argued that permitting the parameter $m>1$ violates the $\beta$-plane approximation and therefore renders invalid the conclusion that the system can be driven unstable. Such an argument would be incorrect since, apart from the directional factor $\cos \phi$ of the wave, the crucial quantity is $\beta V / f^{2}$, or explicitly,

$\frac{\beta V}{f^{2}}=\frac{\cos \theta_{o}}{\sin ^{2} \theta_{o}} \frac{1}{2 M}$.

This number cannot be arbitrarily assigned values very much less than unity, as is normally assumed to give mode frequencies which are well separated (Gill, 1982), since it is determined completely by the orientation of the wave, the latitude of the $\beta$-plane and the equatorial rotational Mach number, which defines the properties of the body. Therefore if we ac- cept the predictions of the dispersion equation for $m<1$ (and also $m \ll 1$ ) we must also accept those for $m>1$ since the solutions for $\omega$ span the same values of $k$. Hence the instability is real and not a consequence of merely arbitrarily allowing $m$ to exceed unity. As we have already indicated, however, equatorial regions do require special treatment (not provided here) but already given by Cane and Sarachik (1976) and Moore and Philander (1977). In conclusion it is possible that the evolution of the instability of these coupled waves may prove important in the nonlinear dynamics of the atmospheres of the planets (and possibly of the Earth's oceans).

Acknowledgements. JFM expresses thanks to the National Research Foundation (South Africa) and the Pei-Ling Chan Chair of Physics (UAH) for their support.

Topical Editor C. Jacobi thanks two anonymous referees for their help in evaluating this paper.

\section{References}

Cane, M. A. and Sarachik, E. S.: Forced baroclinic ocean motions: I. The linear equatorial unbounded case, J. Mar. Res., 34, 629665, 1976.

Charney, J. G. and Drazin, P. G.: Propagation of planetary-scale disturbances from the lower into the upper atmosphere, J. Geophys. Res., 66, 83-109, doi:10.1029/JZ066i001p00083, 1961.

Eckart, C.: Hydrodynamics of Oceans and Atmospheres, Pergamon Press, 1960. 
Gill, A. E.: Atmosphere Ocean Dynamics, vol. 30 of International Geophysics Series, 1982.

Lighthill, J.: Waves in Fluids, Cambridge University Press, 1980. Mekki, O. M. and McKenzie, J. F.: The Propagation of Atmospheric Rossby Gravity Waves in Latitudinally Sheared Zonal Flows, Roy. Soc. London Phil. T. Ser. A, 287, 115-143, 1977.

Moore, D. W. and Philander, S. G. H.: The Sea - Modelling of the Tropical Oceanic Circulation, vol. 6, Wiley - Interscience, 1977.
Pedlosky, J. P.: Geophysical Fluid Dynamics, Springer Verlag, 1987.

Sakai, S.: Rossby-Kelvin instability: a new type of ageostrophic instability caused by a resonance between Rossby waves and gravity waves, J. Fluid Mech., 202, 149-176, doi:10.1017/ S0022112089001138, 1989.

Stoker, J. J.: Water Waves: The Mathematical Theory with Applications, Interscience Publishers, Inc. New York, 1965. 